\title{
A Generalization of a Result of Newman on Multipliers of Difference Sets
}

\author{
R. L. McFarland ${ }^{1}$
}

(June 17, 1965)

\begin{abstract}
A theorem of $\mathrm{M}$. Newman states that if $v, k, \lambda$, are the parameters for a difference set $D$, and $k-\lambda=p$ or $2 p$ ( $p$ a prime) then $p$ is a multiplier of $D$. This theorem is generalized to the case of an abelian difference set and several consequences are noted.
\end{abstract}

Key Words: Abelian, multipliers, block designs, difference sets.

\section{Introduction}

A difference set with parameters $(v, k, \lambda, n)$ is a subset

$$
D=\left\{d_{1}, \ldots ., d_{k}\right\}
$$

of $k$ distinct elements of a (multiplicative) group $G$ with finite order $v$, such that every nonidentity element $g$ in $G$ can be expressed in exactly $\lambda$ ways as

$$
d_{i}^{-1} d_{j}=g, \quad 1 \leqq i, j \leqq k .
$$

The parameter $n$ is defined by

$$
n=k-\lambda \text {. }
$$

Counting the total number of nonidentity "differences," $d_{i}^{-1} d_{j}$, in two ways yields

$$
k(k-1)=\lambda(v-1) .
$$

The difference set $D$ is said to be abelian (cyclic) in case the group $G$ is abelian (cyclic). The exponent, $v^{*}$, of the difference set $D$ is the least common multiple of the orders of the elements of $G$. An integer $t$ is a multiplier of the difference set

$$
D=\left\{d_{1}, \ldots, d_{k}\right\}
$$

in case the sets

$$
\begin{aligned}
& D(t)=\left\{d_{1}^{t}, \ldots, d_{k}^{t}\right\} \\
& g D=\left\{g d_{1}, \ldots, g d_{k}\right\}
\end{aligned}
$$

are identical, apart from order, for some group element $g$ in $G$.

\footnotetext{
'Present address: 6970th Support Group. Fort George Meade. Maryland. 20755
}

Newman $[5]^{2}$ has proved the following result.

THEOREM 1. Let $\mathrm{D}$ be a cyclic difference set with parameters $(\mathrm{v}, \mathrm{k}, \lambda, \mathrm{n})$. Suppose

$$
\mathrm{n}=2 \mathrm{p},(7 \mathrm{p}, \mathrm{v})=1
$$

where $\mathrm{p}$ is a prime. Then $\mathrm{p}$ is a multiplier of $\mathrm{D}$.

Theorem 1 can be generalized in two ways. First of all, it can be generalized to abelian difference sets. Secondly, as H. B. Mann has pointed out, theorem 1 can be combined with the following multiplier theorem.

TheOREM 2. Let D be an abelian difference set with parameters $(\mathrm{v}, \mathrm{k}, \lambda, \mathrm{n})$ and exponent $\mathrm{v}^{*}$. Suppose

$$
\mathrm{n}_{1} \mid \mathrm{n}, \quad\left(\mathrm{n}_{1}, \mathrm{v}\right)=1, \quad \mathrm{n}_{1}>\lambda, \quad \mathrm{n}_{1}=\mathrm{p}_{1}^{\mathrm{e}_{1}} \cdots \mathrm{p}_{\mathrm{s}}^{\mathrm{e}} \mathrm{s}
$$

where the $\mathrm{p}_{1}$ are distinct primes. If there exist integers $\mathrm{f}_{1}, \ldots, \mathrm{f}_{\mathrm{s}}$ such that

$$
\mathrm{t} \equiv \mathrm{p}_{1}^{\mathrm{f}_{1}} \equiv \cdots \cdot \ldots \mathrm{p}_{\mathrm{s}}^{\mathrm{f}_{\mathrm{s}}}\left(\bmod \mathrm{v}^{*}\right)
$$

then $\mathrm{t}$ is a multiplier of $\mathrm{D}$.

Theorem 2 was proven for cyclic difference sets by Hall [2]. It was generalized to abelian difference sets by Menon [4]. More recently, Mann [3] has given another proof of theorem 2 .

These two generalizations of theorem 1 yield:

Theorem 3. Let $\mathrm{D}$ be an abelian difference set with parameters $(\mathrm{v}, \mathrm{k}, \lambda, \mathrm{n})$ and exponent $\mathrm{v}^{*}$. Suppose

$$
\mathrm{n}=2 \mathrm{n}_{1}, \quad\left(7 \mathrm{n}_{1}, \mathrm{v}\right)=1, \quad \mathrm{n}_{1}=\mathrm{p}_{1}^{e_{1}} \ldots \mathrm{p}_{\mathrm{s}}^{e_{\mathrm{s}}}
$$

where the $\mathrm{p}_{\mathrm{i}}$ are distinct primes. If there exist integers $\mathrm{f}_{1}, \ldots, \mathrm{f}_{\mathrm{s}}$ such that

$$
\mathrm{t} \equiv \mathrm{p}_{1}^{\mathrm{f}} 1 \equiv \cdot \cdots \equiv \mathrm{p}_{\mathrm{S}}^{\mathrm{f}_{\mathrm{S}}}\left(\bmod \mathrm{v}^{*}\right)
$$

then $\mathrm{t}$ is a multiplier of $\mathrm{D}$.

\footnotetext{
${ }^{2}$ Figures in brackets indicate the literature references at the end of this paper.
} 
A special case of theorem 3 is worthy of note.

COROLlaRY. Let $\mathrm{D}$ be an abelian difference set with parameters $(\mathrm{v}, \mathrm{k}, \lambda, \mathrm{n})$. If

$$
\mathrm{n}=2 \mathrm{p}^{\mathrm{e}}, \quad \mathrm{e} \geqq 1, \quad(7 \mathrm{p}, \mathrm{v})=1
$$

where $\mathrm{p}$ is a prime, then $\mathrm{p}$ is a multiplier of $\mathrm{D}$.

This paper is devoted to the proof of theorem 3 .

\section{Preparatory Lemmas}

Let $R_{G}$ denote the group ring of the finite multiplicative abelian group $G$ over the rational integers. The elements of $R_{G}$ are of the form

$$
\sum_{g \in G} a_{g} g
$$

where the coefficients $a_{g}$ are integral. Addition in $R_{g}$ is component addition of the coefficients

$$
\sum_{g} a_{g} g+\sum_{g} b_{g} g=\sum_{g}\left(a_{g}+b_{g}\right) g .
$$

Multiplication in $R_{\text {( }}$ is the usual multiplication in an associative algebra with basis consisting of the elements of $G$

$$
\left(\sum_{g} a_{g} g\right)\left(\sum_{\bar{g}} b_{\bar{g}} \bar{g}\right)=\sum_{h} h \sum_{g \bar{g}=h} a_{g} b_{\bar{g}} .
$$

No confusion will result if we let $G$ denote the element

$$
G=\sum_{g} g
$$

in $R_{G}$ that has every coefficient equal to one. Similarly, if the difference set $D$ in $G$ consists of the $k$ elements $d_{1}, \ldots, d_{k}$ we shall write $D$ to denote the element

$$
D=d_{1}+\cdots+d_{k}
$$

in $R_{G}$. For any integer $t$ and any group element $g$ we define

$$
\begin{gathered}
D(t)=d_{1}^{t}+\cdots+d_{k}^{t} \\
t D=t d_{1}+\cdots+t d_{k} \\
g D=g d_{1}+\cdots+g d_{k} .
\end{gathered}
$$

The definition of a difference set implies that

$$
D(-1) D=n+\lambda G
$$

where we have surpressed the identity element of $G$ on $n$. Also

$$
D G=k G .
$$

The integer $t$ is a multiplier of the difference set $D$ if and only if

$$
D(t)=g D
$$

for some $g$ in $G$.

Lemma 1. Let $\mathrm{D}$ and $\mathrm{D}^{*}$ be difference sets with parameters $(\mathrm{v}, \mathrm{k}, \lambda, \mathrm{n})$ in the same group $\mathrm{G}$. Let

$$
\mathrm{F}=\mathrm{D}(-1) \mathrm{D}^{*}-\lambda \mathrm{G} \text {. }
$$

Then

$$
\begin{array}{ll}
\text { (i) } & \mathrm{FG}=\mathrm{nG} \\
\text { (ii) } \mathrm{F}(-\mathrm{l}) \mathrm{F}=\mathrm{n}^{2} \\
\text { (iii) } \mathrm{FD}=\mathrm{nD} *
\end{array}
$$

If $\mathrm{F}$ has nonnegative coefficients, then

$$
\mathrm{gD}=\mathrm{D}^{*}
$$

for some $\mathrm{g}$ in $\mathrm{G}$.

Proof. Parts (i), (ii), and (iii) can be verified by straightforward computations using eqs (1), (2), and (3). If $F$ has nonnegative coefficients, then part (ii) implies that $F$ has exactly one nonzero coefficient, say

$$
F=n g, \quad g \in G .
$$

Then part (iii) implies that

$$
g D=D^{*}
$$

as desired.

LEMma 2. Let $\mathrm{G}$ be a finite abelian group with order $\mathrm{v}$ prime to 2 and 7 . Let $\mathrm{E}$ be an element in the group ring $\mathrm{R}_{\mathrm{G}}$ such that

$$
\begin{aligned}
& \text { (i) } \mathrm{EG}=2 \mathrm{G} \\
& \text { (ii) } \mathrm{E}(-1) \mathrm{E}=4 \text {. }
\end{aligned}
$$

Then E has nonnegative coefficients.

Proof. Let

$$
E=\sum_{g \in G_{i}} a_{y} g
$$

with $a_{g}$ integral. Hypothesis (i) implies

$$
\sum_{g} a_{g}=2
$$

while hypothesis (ii) implies

$$
\sum_{g} a_{g}^{2}=4
$$

and

$$
\sum_{g^{-1} \bar{g}=h} a_{g} a_{\bar{g}}=0, \quad h \neq 1
$$


Assume that $E$ has a negative coefficient. Then eqs (5) and (6) imply that $E$ has one coefficient equal to minus one, three coefficients equal to plus one, and the remaining coefficients zero. Thus

$$
E=-w+x+y+z
$$

for distinct group elements $w, x, y, z$ in $G$. Letting $h=w^{-1} x, w^{-1} y, w^{-1} z$ in eq (7) we obtain either two or four nonzero terms in the left-hand sum. Suppose four nonzero terms occur for $h=w^{-1} x$. Then there are exactly three possibilities, namely

$$
\begin{aligned}
& h=w^{-1} x=x^{-1} w=y^{-1} z=z^{-1} y \\
& h=w^{-1} x=x^{-1} y=y^{-1} z=z^{-1} w \\
& h=w^{-1} x=x^{-1} z=y^{-1} w=z^{-1} y .
\end{aligned}
$$

In each of these three possibilities, $h^{4}=1$. Thus $h=1$, since $v$ is odd; a contradiction. Therefore, by symmetry among $x, y, z$, we conclude that exactly two nonzero terms occur in eq (7) for $h=w^{-1} x, w^{-1} y$, $w^{-1} z$. Now eq (7) implies that there are four possible values for each of $w^{-1} x, w^{-1} y, w^{-1} z$.

$$
\begin{aligned}
& w^{-1} x=x^{-1} y, x^{-1} z, y^{-1} z, z^{-1} y \\
& w^{-1} y=x^{-1} z, y^{-1} x, y^{-1} z, z^{-1} x \\
& w^{-1} z=x^{-1} y, y^{-1} x, z^{-1} x, z^{-1} y
\end{aligned}
$$

We shall use the symbol " $\leftrightarrow$ " to indicate that a contradiction has been obtained.

Case I: $\quad w^{-1} x=x^{-1} y$.

Case $\mathrm{I}_{\mathrm{A}}: \quad w^{-1} x=x^{-1} y, w^{-1} z=x^{-1} y$.

Then $x^{2}=w y=x z \Rightarrow x=z \leftrightarrow$.

Case $\mathrm{I}_{\mathrm{B}}: \quad w^{-1} x=x^{-1} y, w^{-1} z=y^{-1} x$.

Then $w^{-1} x=x^{-1} y=z^{-1} w$ so eq (7) contains more than two nonzero terms $\leftrightarrow$. .

Case $\mathrm{I}_{\mathrm{C}}: \quad w^{-1} x=x^{-1} y, w^{-1} z=z^{-1} y$.

Then $x^{2}=w y=z^{2} \Rightarrow x=z \leftrightarrow$.

Case $\mathrm{I}_{\mathrm{D}}: w^{-1} x=x^{-1} y, w^{-1} z=z^{-1} x$.

Applying the permutation $x \rightarrow z, y \rightarrow x, z \rightarrow y$ to Cases $\mathrm{I}_{\mathrm{A}}, \mathrm{I}_{\mathrm{B}}, \mathrm{I}_{\mathrm{C}}$ we see that $w^{-1} z=z^{-1} x$ implies $w^{-1} y \neq z^{-1} x$, $x^{-1} z, y^{-1} x$. Therefore $w^{-1} x=x^{-1} y$ implies that $w^{-1} z=$ $z^{-1} x$ and $w^{-1} y=y^{-1} z$. Eliminating $w^{-1}$ among these three equations yields $x^{-2} y=y^{-2} z=z^{-2} x$. Now eliminating an appropriate power of $x$ yields $y^{7}=z^{7}$. Thus $y=z$, since $(v, 7)=1 ;$ a contradiction.

Therefore Case I is impossible. By the symmetry among $x, y, z$ we have

$$
\begin{aligned}
& w^{-1} x \neq x^{-1} y, x^{-1} z \\
& w^{-1} y \neq y^{-1} z, y^{-1} x \\
& w^{-1} z \neq z^{-1} x, z^{-1} y .
\end{aligned}
$$

Thus there are eight possibilities remaining, namely

$$
\begin{aligned}
& w^{-1} x=y^{-1} z, z^{-1} y \\
& w^{-1} y=x^{-1} z, z^{-1} x \\
& w^{-1} z=x^{-1} y, y^{-1} x .
\end{aligned}
$$

By the symmetry between $y$ and $z$ we can assume

$$
w^{-1} x=y^{-1} z \text {. }
$$

Either $w^{-1} z=x^{-1} y$, or else $w^{-1} z=y^{-1} x$. In the first case

$$
w^{-1} x=y^{-1} z=w x^{-1} \Rightarrow w^{2}=x^{2} \Rightarrow \mathrm{w}=x \leftrightarrow,
$$

while in the second case

$$
w^{-1} y=x^{-1} z=w y^{-1} \Rightarrow w^{2}=y^{2} \rightarrow w=y \leftrightarrow .
$$

We have now exhausted all possibilities. Therefore $E$ cannot have a negative coefficient, and the proof is complete.

LEMMA 3. Let $\mathrm{D}$ be an abelian difference set with parameters $(\mathrm{v}, \mathrm{k}, \lambda, \mathrm{n})$ in the group $\mathrm{G}$. Let $\mathrm{p}$ be a prime such that

$$
\mathrm{p}^{\mathrm{e}} \mid \mathrm{n}, \quad(\mathrm{p}, \mathrm{v})=1 .
$$

Then for every integer $\mathrm{f}$, the coefficients of

$$
\mathrm{F}=\mathrm{D}(-1) \mathrm{D}\left(\mathrm{p}^{\mathrm{f}}\right)-\lambda \mathrm{G}
$$

are divisible by $\mathrm{p}^{\mathrm{e}}$.

PRoOf. Lemma 3 appears as a part of the proof of corollary 4.1 of Mann [3]. Alternatively, lemma 3 is a special case of eq (3.9) of Menon [4].

\section{Theorem}

THEOREM 3. Let D be an abelian difference set with parameters $(\mathrm{v}, \mathrm{k}, \lambda, \mathrm{n})$ and exponent $\mathrm{v}^{*}$. Suppose

$$
\mathrm{n}=2 \mathrm{n}_{1}, \quad\left(7 \mathrm{n}_{1}, \mathrm{v}\right)=1, \quad \mathrm{n}_{1}=\mathrm{p}_{1}^{\mathrm{e}_{1}} \cdots \mathrm{p}_{\mathrm{s}}^{\mathrm{e}} \mathrm{s}
$$

where the $\mathrm{p}_{\mathrm{i}}$ are distinct primes. If there exist integers $\mathrm{f}_{1}, \ldots, \mathrm{f}_{\mathrm{s}}$ such that

$$
\mathrm{t} \equiv \mathrm{p}_{1}^{\mathrm{f}_{1}} \equiv \cdots \equiv p_{\mathrm{s}}^{\mathrm{f}_{\mathrm{s}}}\left(\bmod \mathrm{v}^{*}\right)
$$

then $\mathrm{t}$ is a multiplier of $\mathrm{D}$.

Proof. If $D$ is a difference set in the group $G$, then $G-D$ is also a difference set. Clearly any multiplier of $D$ is also a multiplier of $G-D$. Furthermore, one of these difference sets has $\lambda>n$. Consequently, if $n_{1}$ is even, then theorem 3 is a special case of theorem 2. Now assume $n_{1}$ is odd. Then $n=2 n_{1}$ is not a square. In this case it is well known (e.g. theorem 3 of Chowla and Ryser [1]) that $v$ is odd. 
Let

$$
F=D(-1) D(t)-\lambda G
$$

Since

$$
D\left(p_{i}^{f_{i}}\right)=D(t), \quad(i=1, \ldots, s)
$$

lemma 3 implies that the coefficients of $F$ are divisible by $n_{1}$. Let

$$
E=n_{1}^{-1} F
$$

Parts (i) and (ii) of lemma 1 imply that $E$ satisfies the hypotheses (i) and (ii) of lemma 2. Therefore $E$, and consequently $F$, has nonnegative coefficients. Then by lemma 1 ,

$$
g D=D(t)
$$

for some group element $g$. Thus $t$ is a multiplier of $D$.

\section{References}

[1] S. Chowla and H. J. Ryser, Combinatorial problems, Can. J. Math. 2, 93-99 (1950).

[2] Marshall Hall, Jr., A survey of difference sets, Proc. Amer. Math. Soc. 7, 975-986 (1956).

[3] H. B. Mann, Balanced incomplete block designs and abelian difference sets, Ill. J. Math. 8, 252-261 (1964).

[4] P. Kesava Menon, Difference sets in abelian groups, Proc. Amer. Math. Soc. 11, 368-376 (1960).

[5] Morris Newman, Multipliers of difference sets, Can. J. Math. 15, 121-124 (1963).

(Paper 69B4-161) 